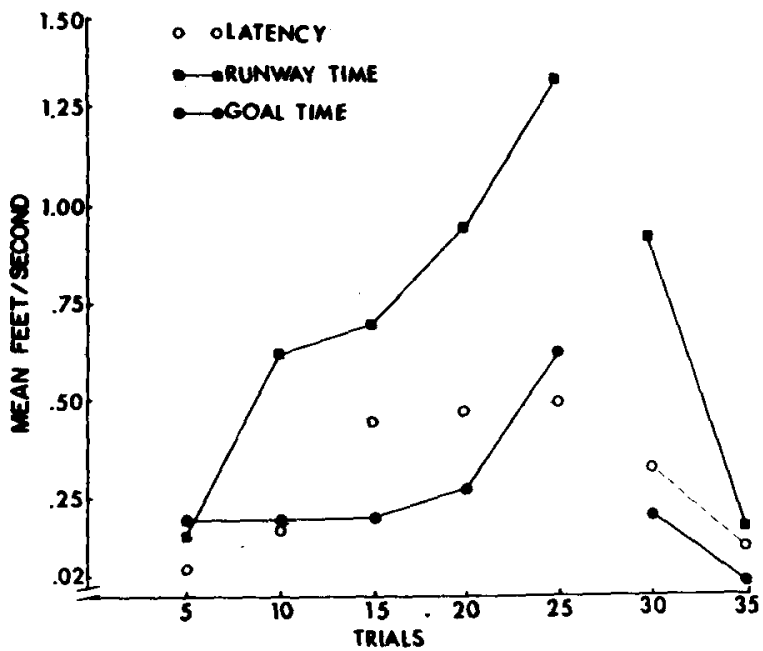

Fig. 1. Acquisition and extinction in the runway.

of four in a food-baited box for $3 \mathrm{~min}$ followed by a further placement in the box singly for $2 \mathrm{~min}$. This procedure results in immediate eating on later occasions when Ss are hungry and alone. The problem of stimulating chicks to move out of a start box was solved by light-proofing the start box and the runway and placing a lamp in or above the goal box, so that this became the only source of light. If light enters the runway from any other source it is unlikely that chicks will proceed beyond that point.

Starting at 4 days of age a limited food access of 6 in $24 \mathrm{~h}$ was sufficient to produce rapid and prolonged eating when food was made available without adversely affecting health. During deprivation is was necessary to keep illumination in the brooder to a low level to prevent cannibalism.

The runway was satisfactory for chicks ranging in age from 4 days to 4 weeks. It was made of aluminum painted flat gray with ceilings of Perspex with fitted, light-proof covers for the start box and runway. The start box measured 12 in $\times 9$ in. $H \times 8$ in. A guillotine door provided access to a runway $3 \frac{1 / 2}{\mathrm{ft}} \times 9 \mathrm{in}$. $\mathrm{H} \times 6$ in. The goal box measured 15 in. $\times 9$ in. $H \times 10$ in. and an electrically operated swing door closed behind $S$ as he entered. The food holder was an aluminum trough 3 in. $\times 2$ in. $H \times 2$ in. The food reward was Chicken Starter, a dry mixture. Ss were permitted to eat from the trough for $3 \mathrm{sec}$ at the end of each trial.

Two treadle-type microswitches in the runway and one photocell lamp in the goal box were used for triggering timers. The treadle switches consisted of two metal plates 1 in. wide with a normally open contact; when $S$ stepped on the plate the contact closed. The latency timer started when the goal box door opened and stopped when $S$ trod on the first switch situated 3 in. inside the runway. This switch also started the second timer that provided a runway measure. The second switch, 3 in. from the end of the runway, stopped the runway timer and started the goal box timer. The photocell was situated directly above the food trough so that $\mathrm{S}$ broke the beam when he placed his head into the trough.

Data were obtained with 12 Australorp chicks obtained at 1 day of age from the Wallaceville Animal Research Station, Wellington. A similar method was used with White Leghorn chicks (Dutch, 1968). The Ss were maintained in a temperaturecontrolled cage-type brooder, with food and water ad lib. After 14 days food was restricted to $6 \mathrm{~h}$ in 24 and $\mathrm{Ss}$ were handled daily for 5 days; on the 6 th day they were fed for $3 \mathrm{~min}$ in a box in groups of four. On Day 7 the procedure was repeated for individual chicks with 2-min feedings.

During acquisition each $S$ was given 5 massed trials per day for 5 days. This was followed by 10 massed extinction trials on Day 6. For the acquisition phase $S$ was placed in the start box and $5 \mathrm{sec}$ later the door was opened. The door was closed when $S$ entered the goal box. The chick was kept in the box for $4 \mathrm{sec}$ and was then carried back to the start box for the next trial. At the end of each set of daily trials, $S$ was placed in a box for $30 \mathrm{~min}$ before being returned to the brooder. The procedure for extinction was the same as for acquisition except that the food trough was empty.

Figure 1 shows the latency, runway, and goal entry times in mean feet per second. Runway time was the most sensitive measure of performance.

\section{REFERENCES}

DUTCH, J. Partial reinforcement and response reversal in chicks. Psychological Reports, 1968, 22, 272-274.

GRINDLEY, G. C. Experiments on the influence of the amount of reward on leaming in young chicks. British Journal of Psychology, 1929, 20 173-180.

\title{
Measurement of electroconvulsive shock intensity
}

\author{
ROBERT R. PAGANO, UNIVERSITY OF WASHINGTON, \\ Seattle, Washington 98105
}

This paper discusses the error introduced in administering ECS by calibrating intensity on the $S$ 's dc rather than ac resistance. Since the ac resistance of rats and mice can be lower than their corresponding $d c$ resistance by a factor of 50, calibrating on $d c$ resistance can introduce appreciable error depending on the current regulation of the ECS device. Examples are included to illustrate the range of possible error. In addition, an alternative method for measuring ECS intensity is presented that greatly reduces measurement error including those due to between- animal resistance differences that necessarily occur when using the usual methods.

It has come to our attention that several laboratories utilizing electroconvulsive shock (ECS) have been reporting ECS intensity based on the animal's dc resistance. However, since most researchers administer ac ( $60 \mathrm{cps}$, sinusoidal) ECS, it is the animal's ac not $\mathrm{dc}$ resistance 2 that will influence the ECS intensity. Since the ac resistance at $60 \mathrm{cps}$ is quite less than the $\mathrm{dc}$, calibrating on $\mathrm{dc}$ resistance can result in administering and reporting intensity levels that are considerably different from the actual intensity delivered. Since ECS intensity has been shown to 


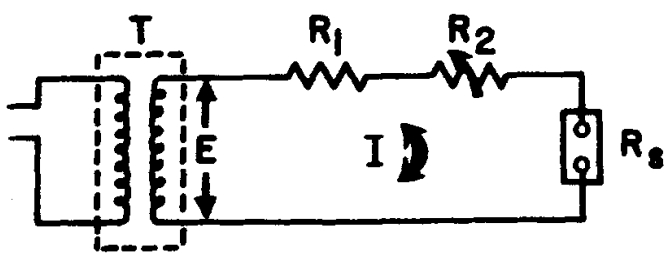

$T=$ Transformer

$R_{1}=$ Resistance of fixed series resistor

$R_{2}=$ Resistance of variable series resistor

\section{$E$ = RMS voltage delivered by secondary of Transformer $T$}

\section{$I$ = RMS current flowing through subject}

\section{$R_{S}=$ Resistance of subject}

Fig. 1. Schematic diagram of typical ECS device.

be an important variable particularly in memory research (Pagano, Bush, Martin, \& Hunt, 1969), errors due to inaccurate intensity reporting can lead to lack of replication and confusion.

The amount of error introduced by dc calibration will depend on two factors: (1) the difference between the animal's ac and dc resistance, and (2) the current regulation of the ECS device. Concerning the first factor, using rats with earclips and mice with corneal electrodes for administering ECS, we have found that the dc resistance varies from about $25 \mathrm{~K}$ to $50 \mathrm{~K}$ depending on specie and electrode configuration. On the other hand, the ac resistance varies between $1.5 \mathrm{~K}$ and $10 \mathrm{~K} .^{3}$

In order to understand the magnitude of possible error introduced by using the animal's dc resistance as the basis for reporting intensity, consider Fig. 1, in which the schematic diagram of a typical ECS device is drawn. Assume $E=1500 \mathrm{~V}$; rats are the Ss and we desire to administer ECS at $40 \mathrm{~mA}$ (rms) for $0.2 \mathrm{sec}$. Also, assume that we adjust $R_{2}$ to give $40 \mathrm{~mA}$ based on the dc resistance of the rat $\left(R_{s}=25 K\right)$.

From Ohm's Law:

$$
\begin{aligned}
I & =\frac{E}{R_{1}+R_{2}+R_{S}} \\
R 1+R 2 & =\frac{E}{I}-R_{s} \\
& =12.5 \mathrm{~K}
\end{aligned}
$$

This leaves us with a circuit where $R_{1}+R_{2}=12.5 \mathrm{~K}$, $\mathrm{E}=1500 \mathrm{~V}$, and we want the rat to get a $40 \mathrm{~mA}$ ECS. However, since we are delivering an ac current, it is the rat's ac resistance rather than dc that will determine the magnitude of current he actually receives. If we assume his ac resistance is $2 K\left(R_{S}=2 K\right)$ the current received is

$$
I=\frac{E}{R I+R 2+R S}=103 \mathrm{~mA}
$$

In this situation the rat will actually be receiving $103 \mathrm{~mA}$ rather than the desired $40 \mathrm{~mA}$. An error of over $150 \%$ has resulted.

The error magnitude will also depend on the current regulation of the ECS device. This, of course, depends on the magnitude of resistance $\left(R_{1}+R_{2}\right)$ in series with the $S$. Consider Fig. 1 again, but this time assume we are using mice and we desire to administer $15 \mathrm{~mA}$ of the comeal ECS. Assume further that our calibration is based on the dc resistance $\left(R_{s}=25 K\right)$ and that $E=1500 \mathrm{~V}$.

From Ohm's Law

$$
\mathbf{R}_{1}+\mathbf{R}_{2}=\frac{E}{I}-R_{s}=75 K
$$

In this situation, since we need much less current, $R_{1}+R_{2}$ can be much larger. This results in a circuit where $R_{1}+R_{2}=75 \mathrm{~K}$ $E=1500 \mathrm{~V}$; we desire the mouse to get $15 \mathrm{~mA}$. Note the amount actually received. Again assume the $S$ 's ac resistance is $2 \mathrm{~K}$ $\left(R_{s}=2 X\right)$.

From Ohm's Law

$$
I=\frac{E}{R_{1}+R_{2}+R_{s}}=19.5 \mathrm{~mA} .
$$

In this case, with a much larger series resistance, the error has been reduced to about $30 \%$. Obviously, the larger the series resistance, the better the current regulation with respect to changes in the S's resistance.

The standard procedure in most laboratories for determining ECS intensity appears to be via calibration with an equivalent resistor. Since animals differ in ac resistance, a better procedure is to measure the current delivered to each animal on line by using a low inertia voltage measuring device such as a polygraph or oscilloscope. By inserting a precision resistor in series with the $S$ and measuring the voltage dropped across this resistor with the polygraph or oscilloscope when ECS is delivered, we can calculate the current flowing through the precision resistor from Ohm's Law. Since the current in a series circuit is everywhere the same, this calculation also gives the current flowing through the $S$.

This technique is used routinely in most laboratories when measuring brain stimulation and has given satisfactory results in our laboratory for measuring ECS.

\section{REFERENCE}

PAGANO, R. R., BUSH D. F., MARTIN, G., \& HUNT, E. B. Duration of retrograde amnesia as a function of electroconvulsive shock intensity. Physiology and Behavior, in press.

\section{NOTES}

1. This research was supported by the U.S. Public Health Service, Grant No. 13673 to the University of Washington and by the University of Washington Graduate School Research Fund No. 11-0309.

2. Since animals are largely resistive and capacitive, to be technically correct we should speak of S's ac impedance, rather than ac resistance. However, since our purpose is to achieve accurate current intensity measurements, without considering phase relationships, we will use the term ac resistance. By ac resistance we mean the value of a resistor, which when substituted for the $S$ will cause the same current magnitude to pass through it as would pass through the $S$.

3. I am indebted to Mr. Daniel F. Bush and Mr. Glen K. Martin for taking these measures. 